\title{
DESENVOLVIMENTO DE INDICADOR DE QUALIDADE DE SANEAMENTO BÁSICO URBANO (IQSBU) E APLICAÇÃO EM CIDADES PARANAENSES ${ }^{1}$
}

\author{
Máriam Trierveiler Pereira* \\ Frederico Fonseca da Silva** \\ Marcelino Luiz Gimenes*** \\ Odacir Antonio Zanatta****
}

RESUMO: O abastecimento por água potável, o esgotamento sanitário, a coleta e disposição de resíduos sólidos e a drenagem pluvial urbana são serviços públicos indispensáveis para uma cidade, pois se relacionam diretamente com a saúde coletiva, a qualidade de vida dos cidadãos e a qualidade ambiental. A partir de dados de fácil aquisição pretendeu-se conhecer a qualidade do saneamento ambiental que o espaço urbano de uma cidade apresentava para que os gestores públicos possam planejar a área urbana de acordo com as necessidades de seus cidadãos e diminuir as deficiências encontradas no saneamento ambiental. O objetivo desse artigo foi desenvolver um Indicador de Qualidade de Saneamento Básico Urbano (IQSBU) e aplicá-lo às cidades paranaenses com mais de 100.000 habitantes, de 2000 a 2008. Para isso, foi necessário: (i) coletar dados sobre saneamento ambiental; (ii) determinar parâmetros limites dos dados escolhidos; (iii) calcular os indicadores de qualidade para cada cidade; e, (iv) comparar os resultados obtidos entre as cidades. Após o término desse estudo, constatou-se a falta de informações sobre o saneamento ambiental urbano, em especial sobre resíduos sólidos e drenagem. Isso implica em dificuldades na gestão e no planejamento urbano. Com relação aos resultados, conclui-se que, no geral, as cidades apresentaram uma boa qualidade de saneamento básico, em pelo menos um ano do período. Observou-se, entretanto, que nenhuma cidade atingiu ótima qualidade dos serviços de saneamento básico $(>80,0)$. As cidades que não tiveram nenhum valor de IQSBU acima de 60 foram: Apucarana, Araucária e Colombo. O ano com melhores resultados de IQSBU e, consequentemente, de maior repasse das informações pelos municípios ao sistema

\footnotetext{
${ }^{1}$ Parte da tese de doutorado da primeira autora.

"Engenheira Civil; Doutora em Engenharia Química; Docente e Pesquisadora do Instituto Federal do Paraná (IFPR), câmpus Umuarama, Brasil; E-mail: mariam.pereira@ifpr.edu.br.

*A Agrônomo; Doutor em Irrigação e Meio Ambiente; Docente e Pesquisador do Instituto Federal do Paraná (IFPR), câmpus Curitiba, Brasil.

${ }^{* * *}$ Engenheiro Químico; Pós-doutor em Engenharia Química; Docente e Pesquisador da Universidade Estadual de Maringá (UEM), Brasil.

**** Agrônomo, Doutor em Agronomia; Docente e Pesquisador do Instituto Federal do Paraná (IFPR), câmpus Umuarama, Brasil.
} 
foi 2006. Nesse ano, o maior valor encontrado para IQSBU foi para Londrina $(78,3)$ e o menor foi para Colombo $(49,8)$.

PALAVRAS-CHAVE: Gestão Ambiental; Índice; Planejamento Urbano.

\section{DEVELOPMENT OF QUALITY INDEX IN BASIC URBAN SANITATION AND ITS APPLICATION IN THE TOWNS OF THE STATE OF PARANÁ, BRAZIL}

ABSTRACT: Drinkable water supply, sewerage, the collection and disposal of solid wastes and urban rain drains are indispensible public services in towns and cities that are concerned with the health of the community, life quality and environment. Easily acquired data provided the quality of environmental sanitation that the town or city offered so that government administrators could plan the urban area according to the needs of its citizens and decrease the deficiencies in environmental sanitation. Current assay developed a Quality Index of Basic Urban Sanitation to be applied to cities with over 100,000 inhabitants in the state of Paraná, Brazil, between 2000 and 2008. (1) The collection of data on environmental sanitation, (2) the determination of limit parameters of chosen data; (3) the calculation of quality indicators for each city; (4) the comparison of results from cities were required. The study registered a dire lack of information on urban environmental sanitation, especially on solid residues and sewerage. The above implies difficulties with regard to administration and urban planning. Results show that, as a rule, the cities under analysis offered a rather good basic sanitation within at least one year. However, no city had the best quality in basic sanitation service $(>80.0)$. The cities with no quality rate above 60 comprised Apucarana, Araucária and Colombo. The year 2006 was the best for Quality Index information and, consequently, for the highest transmission of information by the municipalities to the system. During 2006 the highest Quality Index rate belonged to Londrina (78.3) and the lowest to Colombo (49.8).

KEY WORDS: Environmental Administration; Index; Urban Planning.

\section{INTRODUÇÃO}

O abastecimento por água potável, o esgotamento sanitário, a coleta e disposição de resíduos sólidos e a drenagem pluvial urbana são serviços públicos 
indispensáveis para uma cidade, pois se relacionam diretamente com a saúde coletiva, a qualidade de vida dos cidadãos e a qualidade ambiental.

Existem várias doenças de veiculação hídrica que podem ser evitadas com o fornecimento de água tratada, assim como com a correta coleta e tratamento do esgoto doméstico, como diarréia, cólera, tifo, hepatite e outras.

Da mesma forma, a decomposição dos resíduos sólidos orgânicos provoca o aparecimento de vetores de doenças, como baratas, ratos, mosquitos etc.

Já a drenagem urbana é importante por evitar alagamentos, enchentes, inundações e deslizamentos de encostas. São inúmeros e severos os danos causados por esses desastres de eventos críticos hidrológicos, como mortes, doenças, perdas de habitações, veículos, vias, infraestruturas e outros.

Em vista do exposto, o objetivo desse artigo foi desenvolver um Indicador de Qualidade de Saneamento Básico Urbano (IQSBU) e aplicá-lo às cidades paranaenses com mais de 100.000 habitantes. Para isso, foi necessário: (i) coletar dados sobre saneamento ambiental; (ii) determinar parâmetros limites dos dados escolhidos; (iii) calcular os indicadores de qualidade para cada cidade; e, (iv) comparar os resultados obtidos entre as cidades.

Pretendeu-se, com a realização desta pesquisa, saber, a partir de dados de fácil aquisição, qual era a qualidade do saneamento ambiental que o espaço urbano de uma cidade apresentava. Com essas informações, os gestores públicos têm como planejar o espaço urbano de acordo com as necessidades de seus cidadãos e diminuir as deficiências encontradas no saneamento ambiental.

\section{FUNDAMENTAÇÃO TEÓRICA}

\subsection{INDICADORES E ÍNDICES}

Existe certa confusão sobre o significado de indicadores e índices, onde erroneamente são utilizados como sinônimos (SICHE et al., 2007). Os índices são valores dados a indicadores definidos como importantes no processo de interação homem-meio ambiente, homem-economia, economia-meio ambiente (MAIA et al., 
2001). Para se obter índices, é necessário construir indicadores, que por sua vez são construídos com dados analisados a partir de um conjunto de dados primários, como mostra a Figura 1.

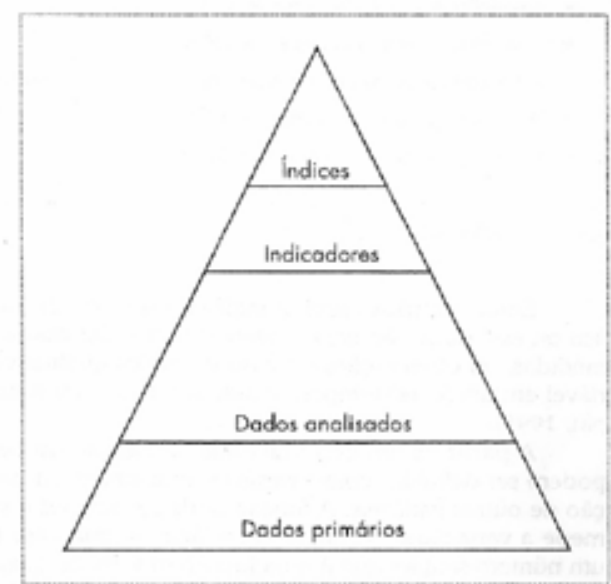

Figura 1. Pirâmide de informações

Fonte: Hammond et al. (1995 apud Bellen, 2006).

A Organização de Cooperação e Desenvolvimento Econômico (OCDE, 1993) define como indicador um parâmetro ou valor derivado de parâmetros que fornecem informações sobre o estado do ambiente. Assim, os indicadores finais são formados por agregação de dados primários, ou de dados analisados ou, ainda, por indicadores primários (BOLLMANN, 2001). O objetivo principal dos indicadores é possibilitar a comparação entre informações em um dado intervalo de tempo (HOLDEN, 2006).

Para Deakin et al. (2007), as principais funções dos indicadores são, entre outras, a avaliação de condições e tendências, e a comparação entre lugares e situações, onde bons indicadores devem, entre outros aspectos, possuir ampla base de dados com fácil acesso, devem ser claros nos valores e em seu conteúdo, devem ser relevantes politicamente e suficientes para uma ação política, e devem ser provocativos, levando à discussão, ao aprendizado e à mudança. 


\subsection{SANEAMENTO BÁSICO URBANO}

Entende-se por saneamento básico urbano o abastecimento público de água; o esgotamento sanitário; a coleta, tratamento e disposição final dos resíduos sólidos e a drenagem urbana.

Para a verificação da qualidade da água tratada, o Ministério da Saúde publicou a Portaria $n^{0}$ 2.914/2011, que revogou a Portaria $n^{0} 518 / 2004$, que dispóe sobre os procedimentos de controle e de vigilância da qualidade da água para consumo humano e seu padrão de potabilidade.

Alguns parâmetros que devem ser analisados pela companhia de saneamento, segundo a Portaria $n^{0}$ 2.914/2011, seus limites para potabilidade e a frequência de amostragem estão mostrados no Quadro 1.

Quadro 1. Valores máximos permitidos para os parâmetros analisados para potabilidade

\begin{tabular}{|c|c|c|c|}
\hline Parâmetros & $\begin{array}{c}\text { Valor máximo } \\
\text { permitido }\end{array}$ & Valor mínimo & $\begin{array}{c}\text { Frequência de } \\
\text { amostragem }\end{array}$ \\
\hline Coliformes totais & Ausência em $100 \mathrm{~mL}$ & - & 2 por semana \\
\hline $\begin{array}{c}\text { Coliformes } \\
\text { termotolerantes }\end{array}$ & Ausência em $100 \mathrm{~mL}$ & - & 2 por semana \\
\hline Turbidez & $0,5 \mathrm{uT}$ & - & A cada 2 horas \\
\hline Cor aparente & $15 \mathrm{uH}$ & - & A cada 2 horas \\
\hline Cloro Residual Livre & $5 \mathrm{mg} \mathrm{L}^{-1}$ & $0,2 \mathrm{mg} \mathrm{L}^{-1}$ & A cada 2 horas \\
\hline Fluoreto & $1,5 \mathrm{mg} \mathrm{L}^{-1}$ & & A cada 2 horas \\
\hline Trihalometanos totais & $0,1 \mathrm{mg} \mathrm{L}^{-1}$ & - & Trimestral \\
\hline Pesticidas & Variável & - & Semestral \\
\hline
\end{tabular}

Fonte: Portaria $\mathrm{n}^{\circ}$ 518, de 25 de março de 2004.

Dos municípios estudados, a Companhia de Saneamento do Paraná (SANEPAR) não atua apenas em Paranaguá, onde a CAB Águas de Paranaguá é responsável pelo saneamento. Os demais municípios têm $100 \%$ da população abastecida por água tratada pela SANEPAR (SANEPAR, 2008).

Com relação ao esgoto sanitário, deve-se observar o disposto na Resolução CONAMA $n^{\circ}$ 430/2011. No Brasil, segundo o IBGE, em 2008, pouco mais da metade 
dos municípios brasileiros tinha rede coletora de esgotos e apenas $28,5 \%$ dos municípios faziam o tratamento desse esgoto coletado. A região Sul tratava 24,1\% dos esgotos coletados (IBGE, 2011).

De acordo com dados, os vazadouros a céu aberto, conhecidos como "lixões", ainda são o destino final dos resíduos sólidos em 50,8\% dos municípios brasileiros. Esse quadro ainda teve uma mudança significativa nos últimos 20 anos: em 1989, eles representavam o destino final de resíduos sólidos em $88,2 \%$ dos municípios. Paralelamente, houve uma expansão no destino dos resíduos para os aterros sanitários, solução mais adequada, que passou de 17,3\% dos municípios, em 2000, para 27,7\%, em 2008 (IBGE, 2011).

Em todo o país, aproximadamente $26,8 \%$ dos municípios que possuíam serviço de manejo de resíduos sólidos sabiam da presença de catadores nas unidades de disposição final de resíduos sólidos (IBGE, 2011). Esse é um grande problema social enfrentado por gestores urbanos, pois os catadores vivem em condições subhumanas, inclusive crianças.

Uma boa solução para esse problema social, que também resolve um problema ambiental, é a instalação de coleta seletiva nas cidades. O Ministério das Cidades, juntamente com o Ministério do Meio Ambiente, elaboraram, em 2008, um manual para a implantação de coleta seletiva nos municípios (BRASIL, 2008).

Mas, antes mesmo já existiam excelentes exemplos em várias cidades brasileiras. Os programas de coleta seletiva de resíduos sólidos aumentaram de 58 identificados em 1989 para 451 em 2000 e alcançando o patamar de 994 em 2008. Os municípios com serviço de coleta seletiva separavam, prioritariamente, papel e/ ou papelão, plástico, vidro e metal (materiais ferrosos e não ferrosos), sendo que os principais compradores desses materiais eram os comerciantes de recicláveis $(53,9 \%)$, as indústrias recicladoras $(19,4 \%)$, entidades beneficentes $(12,1 \%)$ e outras entidades (18,3\%) (IBGE, 2011).

Com relação à drenagem urbana, verifica-se que as inundações são cada vez mais frequentes, quer seja pela alteração do regime de chuva, quer seja pela urbanização de áreas de risco (margens de rios e encostas). Uma solução para o problema de inundações é o Plano Diretor de Drenagem Urbana, como mostra Tucci (2002). 
De acordo com IBGE (2011), a erosão no perímetro urbano afetava, em 2008, 27,3\% dos municípios brasileiros que faziam manejo de águas pluviais. Entre os principais fatores indicados como causas desse problema estavam as condições geológicas e morfológicas $(47,8 \%)$ e as ocupações intensas e desordenadas do solo (46,4\%).

Dos municípios que informaram ter manejo de águas pluviais, 48,7\% declararam não ter tido problemas com inundações nos últimos cinco anos, nem pontos de estrangulamento no sistema de drenagem. Já 27,4\% informaram condição exatamente oposta: deles, 60,7\% disseram haver ocupação urbana em áreas inundáveis naturalmente por cursos d'água e 48,1\% informaram que havia áreas urbanas irregulares em baixios naturalmente inundáveis (IBGE, 2011).

Um grupo de 1.933 municípios brasileiros (34,7\% do total) relatou ter, em seu perímetro urbano, áreas de risco que demandam drenagem especial. Os municípios declararam que as áreas sem infraestrutura de drenagem (62,6\%) e as áreas de baixios (depressões) sujeitas a inundações e/ou proliferação de transmissores de doenças $(56,8 \%)$ eram os tipos mais encontrados e suscetíveis a riscos no perímetro urbano. No Sudeste, percentual significativo (56\%) era atribuído às áreas em taludes (planos inclinados que limitam aterros) e encostas sujeitas a deslizamentos, configuração geomorfológica peculiar aos estados desta região (IBGE, 2011).

A drenagem também é responsável por diminuir os riscos ambientais de tempestades. Em junho de 2011 ocorreu o $2^{\circ}$ Congresso Mundial de Cidades Resilientes, em Bonn (Alemanha). Foram discutidos os fatores que fazem com que uma cidade seja resiliente, ou seja, que consiga se adaptar após eventos climáticos críticos (ICLEI, 2011).

\section{MATERIAIS E MÉTODOS}

O IQSBU faz parte de um estudo mais amplo para o desenvolvimento de um Indicador de Qualidade Socioambiental Urbana (IQSAU). O IQSAU é composto por quatro dimensões: (i) dimensão socioeconômica, que engloba questões como educação, saúde, segurança, emprego e renda, moradia, cultura e lazer; (ii) dimensão 
de infraestrutura e serviços, que leva em consideração mobilidade e acessibilidade, saneamento ambiental, energia elétrica, comunicações; (iii) dimensão ambiental, resultante da qualidade ambiental da água, do ar, do solo e das áreas verdes; e, (iv) dimensão climática, cujas variáveis são precipitação, temperatura do ar, umidade relativa e velocidade do vento de superfície.

O IQSBU é composto por quatro subindicadores: (i) Indicador de Qualidade de Abastecimento de Água (IQAB); (ii) Indicador de Qualidade de Esgotamento Sanitário (IQES); (iii) Indicador de Qualidade de Coleta e Disposição de Resíduos Sólidos (IQRS); e, (iv) Indicador de Qualidade de Drenagem de Águas Pluviais (IQD).

Os critérios para seleção desses indicadores foi a disponibilidade de dados urbanos anuais que caracterizam cada um dos indicadores. A concepção do IQSBU pode ser visualizada no Quadro 2.

Quadro 2. Concepção do IQSBU

\begin{tabular}{|c|c|c|}
\hline Indicador & Subindicador & Dado ou variável \\
\hline \multirow{16}{*}{$\begin{array}{c}\text { Indicador de } \\
\text { Qualidade } \\
\text { Saneamento Básico } \\
\text { Urbano }\end{array}$} & \multirow{6}{*}{$\begin{array}{c}\text { Indicador de } \\
\text { Qualidade de } \\
\text { Abastecimento de } \\
\text { Água }\end{array}$} & Tarifa média de água \\
\hline & & Consumo médio per capita de água \\
\hline & & Índice de atendimento urbano de água \\
\hline & & Índice de perdas na distribuição \\
\hline & & Duração média das paralisações \\
\hline & & Índice de Conformidade da Água Tratada \\
\hline & \multirow{4}{*}{$\begin{array}{l}\text { Indicador de } \\
\text { Qualidade de } \\
\text { Esgotamento } \\
\text { Sanitário }\end{array}$} & Tarifa média de esgoto \\
\hline & & Índice de coleta de esgoto \\
\hline & & Índice de tratamento de esgoto \\
\hline & & Índice de atendimento urbano de esgoto \\
\hline & \multirow{5}{*}{$\begin{array}{c}\text { Indicador de } \\
\text { Qualidade } \\
\text { de Coleta e } \\
\text { Disposição de } \\
\text { Resíduos Sólidos }\end{array}$} & Taxa de cobertura do serviço de coleta de resíduos \\
\hline & & Custo unitário médio do serviço de coleta \\
\hline & & Taxa de recuperação de materiais recicláveis \\
\hline & & Custo unitário médio do serviço de varrição \\
\hline & & Disposição dos resíduos sólidos \\
\hline & $\begin{array}{l}\text { Indicador de } \\
\text { Qualidade de } \\
\text { Drenagem de } \\
\text { Águas Pluviais }\end{array}$ & Número anual de enchentes ou alagamentos \\
\hline
\end{tabular}


Os municípios paranaenses para aplicação do indicador foram os que contavam com 100.000 habitantes em 2008 e possuíam dados disponíveis de saneamento.

Os dados foram coletados no Ministério das Cidades, disponíveis no Sistema Nacional de Informações sobre Saneamento (SNIS) (MCIDADES, 2009). Neste sistema estão disponíveis mais de 40 dados e indicadores sobre água, esgoto e resíduos sólidos.

Os dados de conformidade de água tratada foram fornecidos pela SANEPAR, em relatórios de 2005 a 2008 (SANEPAR, 2005; SANEPAR, 2006; SANEPAR, 2007; SANEPAR, 2008). Nesses relatórios são apresentadas informações sobre legislação de potabilidade, o sistema de tratamento da água, a bacia de onde a água é captada, os parâmetros analisados, o número de amostras realizadas e o número de amostras atendidas.

Os dados sobre alagamentos e enchentes, de 2005 a 2007, são de relatórios da Defesa Civil (SUDERHSA, 2009b) onde são mostrados os eventos críticos e o número de atingidos por municípios.

Os dados de destino dos resíduos sólidos são da Superintendência de Desenvolvimento de Recursos Hídricos e Saneamento Ambiental (SUDERHSA, 2009a) e do SNIS (MCIDADES, 2009) de 2002 a 2006.

\subsection{INDICADOR DE QUALIDADE DE ABASTECIMENTO DE ÁGUA (IQAB)}

O IQAB reúne as seguintes variáveis: tarifa média de água, consumo médio per capita de água, índice de atendimento urbano de água, índice de perdas na distribuição, duração média das paralisações e índice de conformidade de água tratada.

Os valores para cada variável de uma localidade foram transformados em uma escala de 0 a 100, de acordo com os limites mostrados no Quadro 3, por interpolação simples. 
Quadro 3. Variáveis para o cálculo do IQAB e seus limites

\begin{tabular}{|c|c|c|c|c|}
\hline Códigos & Variáveis & $\begin{array}{l}\text { Limites para } \\
\mathrm{IQAB}=100\end{array}$ & $\begin{array}{c}\text { Limites para } \\
\mathrm{IQAB}=50\end{array}$ & $\begin{array}{l}\text { Limites para } \\
\mathrm{IQAB}=0\end{array}$ \\
\hline $\mathbf{A B}_{1}$ & Tarifa média de água & $\begin{array}{c}\text { valor mínimo } \\
\text { estadual [R } \$ \\
\left.\mathrm{~m}^{3}\right]\end{array}$ & $\begin{array}{l}\text { valor máximo } \\
\text { estadual }\end{array}$ & - \\
\hline $\mathbf{A B}_{2}$ & $\begin{array}{c}\text { Consumo médio per capita de } \\
\text { água }\end{array}$ & $\begin{array}{l}<150 \mathrm{~L} / \mathrm{hab} \\
\mathrm{dia}\end{array}$ & - & $\begin{array}{l}\text { valor máximo } \\
\text { estadual }\end{array}$ \\
\hline $\mathbf{A B}_{3}$ & $\begin{array}{l}\text { Índice de atendimento urbano } \\
\text { de água }\end{array}$ & $100 \%$ & - & $0 \%$ \\
\hline $\mathbf{A B}_{4}$ & Índice de perdas na distribuição & $0 \%$ & - & $100 \%$ \\
\hline $\mathbf{A B}_{5}$ & Duração média das paralisações & $\begin{array}{l}\text { valor mínimo } \\
\text { estadual } \\
\text { [horas/ } \\
\text { paralisação] }\end{array}$ & $\begin{array}{l}\text { valor máximo } \\
\text { estadual }\end{array}$ & - \\
\hline $\mathbf{A B}_{6}$ & $\begin{array}{l}\text { Índice de Conformidade da } \\
\text { Água Tratada (ICAT) }\end{array}$ & $0 \%$ & - & $100 \%$ \\
\hline
\end{tabular}

${ }^{a}$ ICAT - Índice de Conformidade dos parâmetros analisados mensalmente (limites definidos pela Portaria $\mathrm{n}^{0}$ 518/04)

Para as variáveis 'tarifa média de água' e 'duração média das paralisações' adotou-se 50,0 como limite inferior para IQAB quando há valores na base de dados. Dessa forma, se os dados não foram repassados para o Ministério das Cidades, o valor do IQAB é zero. Essa medida privilegia as localidades que enviam seus relatórios ao MCidades.

Os parâmetros de $\mathrm{AB}_{1} \mathrm{a} \mathrm{AB}_{5}$ foram diretamente coletados do Sistema Nacional de Informações sobre Saneamento (SNIS). Para o cálculo do Índice de Conformidade da Água Tratada (ICAT) foram desenvolvidas as equações (1) e (2).

A equação (2) é uma média ponderada levando-se em consideração os parâmetros relacionados à qualidade da água bem como a toxicidade dos compostos analisados.

$$
I C A T_{x, y}=100-I N C_{x, y}
$$

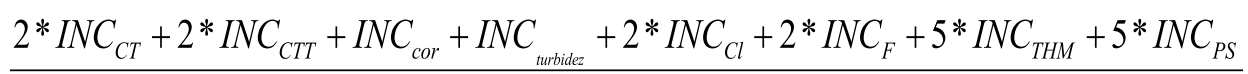


Com:

$\mathbf{x}=$ localidades, de 1 a 16 ;

$\mathrm{y}=$ tempo, de $2000 \mathrm{a} 2008$.

Onde:

ICAT $_{x, y}$ - Índice de Conformidade da Água Tratada na cidade $x$ no ano $y$;

$\mathrm{INC}_{\mathrm{x}, \mathrm{y}}$ - Índice de Não-Conformidade da Água Tratada na cidade $x$ no ano $y$;

CT - análise de coliformes totais na cidade $x$ no ano $y$;

CTT - análise de coliformes termotolerantes na cidade $x$ no ano $y$;

$\mathrm{Cl}$ - análise de cloro na cidade $x$ no ano $y$;

$\mathrm{F}$ - análise de flúor na cidade $x$ no ano $y$;

THM - análise de trihalometanos na cidade $x$ no ano $y$;

PS - análise de pesticidas na cidade $x$ no ano $y$.

Para calcular a não-conformidade de cada parâmetro, foi desenvolvida a equação (3).

$$
I N C_{i}=\frac{\left(100 * \frac{A R_{i}-A A L_{i}}{A R_{i}}\right)+\left(100 * \frac{M N C_{i}}{12}\right)}{2}
$$

Onde:

$i$ é cada parâmetro analisado (CT - coliformes totais; CTT - coliformes termotolerantes; Cl - cloro; F - flúor; THM - trihalometanos; PS pesticidas);

AR - número de amostras realizadas na cidade $x$ no ano $y$;

AAL - número de amostras atendidas pela legislação na cidade $x$ no ano $y$;

MNC - número de meses onde houve amostras não-conformes na cidade $x$ no ano $y$. 
O IQAB foi calculado conforme equação (4).

$$
I Q A B_{x, y}=\frac{A+B+C+D+E+F}{6}
$$

Sendo:

$$
\begin{aligned}
& A=\left\{\left[50 \frac{\left(\text { Tmáx }-A B 1_{x, y}\right)}{\text { Tmáx }- \text { Tmín }}\right]+50\right\} \\
& B=\left[100 \frac{\left(\text { Cmáx }-A B 2_{x, y}\right)}{C m a ́ x-a}\right] \\
& C=A B 3_{x, y} \\
& D=\left(100-A B 4_{x, y}\right) \\
& E=\left\{\left[50 \frac{\left(T P m a ́ x-A B 5_{x, y}\right)}{T P m a ́ x-T P m i ́ n}\right]+50\right\} \\
& F=A B 6_{x, y}
\end{aligned}
$$

Com:

$\mathbf{x}=$ localidades, de 1 a 16 ;

$\mathrm{y}=$ tempo, de 2000 a 2008.

Onde:

$\mathrm{IQAB}_{\mathrm{x}, \mathrm{y}}$ - Indicador de Qualidade de Abastecimento de Água na cidade $x$ no ano $y$;

$\mathrm{AB}_{1 \mathrm{x}, \mathrm{y}}=$ tarifa média de água na cidade $x$ no ano $y$;

Tmáx = tarifa máxima estadual de água no ano $y$;

Tmín = tarifa mínima estadual de água no ano $y$;

$\mathrm{AB}_{2 \mathrm{x}, \mathrm{y}}=$ consumo médio per capita de água na cidade $x$ no ano $y$;

Cmáx = consumo médio máximo estadual no ano $y$; 
Cmín $=$ consumo médio mínimo estadual no ano $y$;

$\mathrm{AB}_{3 \mathrm{x}, \mathrm{y}}=$ índice de atendimento urbano de água na cidade $x$ no ano $y$;

$\mathrm{AB}_{4 \mathrm{x}, \mathrm{y}}=$ índice de perdas na distribuição na cidade $x$ no ano $y$;

$\mathrm{AB}_{5 \mathrm{x}, \mathrm{y}}=$ duração média das paralisações na cidade $x$ no ano $y$;

TPmáx $=$ duração média máxima estadual no ano $y$;

TPmín = duração média mínima estadual no ano $y$;

$\mathrm{AB}_{6 \mathrm{x}, \mathrm{y}}=$ Índice de Conformidade da Água Tratada na cidade $x$ no ano $y$;

$a=150 \mathrm{~L} /$ hab.dia;

\subsection{INDICADOR DE QUALIDADE DE ESGOTAMENTO SANITÁRIO (IQES)}

O IQES reúne as seguintes variáveis: tarifa média de esgoto, índice de coleta de esgoto, índice de tratamento de esgoto, índice de atendimento urbano de esgoto referido aos municípios atendidos com água.

Os valores para cada variável de uma localidade foram transformados em uma escala de 0 a 100, de acordo com os limites mostrados no Quadro 4, por interpolação simples.

Quadro 4. Variáveis para o cálculo do IQES e seus limites

\begin{tabular}{|c|c|c|c|c|}
\hline Códigos & Variáveis & $\begin{array}{c}\text { Limites } \\
\text { para } \\
\text { IQES = 100 }\end{array}$ & $\begin{array}{c}\text { Limites } \\
\text { para } \\
\text { IQES = 50 }\end{array}$ & $\begin{array}{c}\text { Limites } \\
\text { para } \\
\text { IQES = 0 }\end{array}$ \\
\hline $\mathbf{E S}_{1}$ & Tarifa média de esgoto [R $\left.\$ / \mathrm{m}^{3}\right]$ & $\begin{array}{c}\text { valor } \\
\text { mínimo } \\
\text { estadual }\end{array}$ & $\begin{array}{c}\text { valor } \\
\text { máximo } \\
\text { estadual }\end{array}$ & - \\
\hline $\mathbf{E S}_{2}$ & Índice de coleta de esgoto [\%] & $100 \%$ & - & $0 \%$ \\
\hline $\mathbf{E S}_{3}$ & Índice de tratamento de esgoto [\%] & $100 \%$ & - & $0 \%$ \\
\hline $\mathbf{E S}_{4}$ & $\begin{array}{c}\text { Índice de atendimento urbano de } \\
\text { esgoto [\%] }\end{array}$ & $100 \%$ & - & $0 \%$ \\
\hline
\end{tabular}


Para o cálculo do IQES foi desenvolvida a equação (5).

$$
I Q E S_{x, y}=\frac{\left\{\left[50 \frac{\left(T E m a ́ x-E S_{1 x, y}\right)}{T E m a ́ x-T E m i ́ n}\right]+50\right\}+E S_{2 x, y}+E S_{3 x, y}+E S_{4 x, y}}{4}
$$

Com:

$\mathrm{x}=$ localidades, de $1 \mathrm{a} 16$;

$\mathrm{y}=$ tempo, de 2000 a 2008.

Onde:

$\mathrm{IQES}_{\mathrm{x}, \mathrm{y}}$ - Indicador de Qualidade de Esgotamento Sanitário na cidade $x$ no ano $y$;

$\mathrm{ES}_{1 \mathrm{x}, \mathrm{y}}=$ tarifa média de esgoto na cidade $x$ no ano $y$;

TEmáx $=$ tarifa máxima estadual de esgoto no ano $y$;

TEmín $=$ tarifa mínima estadual de esgoto no ano $y$;

$\mathrm{ES}_{2 \mathrm{x}, \mathrm{y}}=$ índice de coleta de esgoto na cidade $x$ no ano $y$;

$\mathrm{ES}_{3 \mathrm{x}, \mathrm{y}}=$ índice de tratamento de esgoto na cidade $x$ no ano $y$;

$\mathrm{ES}_{4 \mathrm{x}, \mathrm{y}}=$ índice de atendimento urbano de esgoto na cidade $x$ no ano $y$;

3.3 INDICADOR DE QUALIDADE DE COLETA E DISPOSIÇÃO DE RESÍDUOS SÓLIDOS (IQRS)

O IQRS reúne as variáveis: taxa de cobertura do serviço de coleta de resíduos em relação à população urbana, custo unitário médio do serviço de coleta, taxa de recuperação de materiais recicláveis (exceto matéria orgânica e rejeitos) em relação à quantidade total coletada, custo unitário médio do serviço de varrição e disposição dos resíduos sólidos.

Os valores para cada variável de uma localidade foram transformados em uma escala de 0 a 100, de acordo com os limites mostrados no Quadro 5, por interpolação simples. 
Quadro 5. variáveis para o cálculo do IQRS e seus limites

\begin{tabular}{|c|c|c|c|}
\hline Códigos & Variáveis & $\begin{array}{c}\text { Limites } \\
\text { para } \\
\text { IQRS = } \\
100\end{array}$ & $\begin{array}{c}\text { Limites } \\
\text { para } \\
\text { IQRS = } 0\end{array}$ \\
\hline $\mathrm{RS}_{1}$ & $\begin{array}{l}\text { Taxa de cobertura do serviço de coleta de resíduos } \\
{[\%]}\end{array}$ & $100 \%$ & $0 \%$ \\
\hline $\mathbf{R S}_{2}$ & Custo unitário médio do serviço de coleta $[\mathrm{R} \$ / \mathrm{t}]$ & $\begin{array}{l}\text { valor } \\
\text { mínimo } \\
\text { estadual }\end{array}$ & $\begin{array}{l}\text { valor } \\
\text { máximo } \\
\text { estadual }\end{array}$ \\
\hline $\mathbf{R S}_{3}$ & Taxa de recuperação de materiais recicláveis [\%] & $50 \%$ & $0 \%$ \\
\hline $\mathbf{R S}_{4}$ & Custo unitário médio do serviço de varrição [R $\$ / K m]$ & $\begin{array}{l}\text { valor } \\
\text { mínimo } \\
\text { estadual }\end{array}$ & $\begin{array}{l}\text { valor } \\
\text { máximo } \\
\text { estadual }\end{array}$ \\
\hline $\mathbf{R S}_{5}$ & Disposição dos resíduos sólidos a & $\begin{array}{l}\text { existência } \\
\text { de aterro } \\
\text { sanitário }\end{array}$ & $\begin{array}{l}\text { existência de } \\
\quad \text { lixão }\end{array}$ \\
\hline
\end{tabular}

${ }^{a}$ Foram consideradas as seguintes disposições: lixão, aterro controlado e aterro sanitário, conforme SUDERHSA (2009a).

Foi desenvolvida a equação (6) para o cálculo do IQRS.

$I Q R S_{x, y}=\frac{R S_{1 x, y}+\left(100 \frac{R S_{2 x, y}-C R S m a ́ x}{C R S m a ́ x-C R S m i ́ n}\right)+2 R S_{3 x, y}+\left(100 \frac{R S_{4 x, y}-C V m a ́ x}{C V m a ́ x-C V m i ́ n}\right)+R S_{5 x, y}}{6}$

Com:

$\mathrm{x}=$ localidades, de 1 a 16 ;

$\mathrm{y}=$ tempo, de $2000 \mathrm{a} 2008$.

Onde:

$\mathrm{IQRS}_{\mathrm{x}, \mathrm{y}}$ - Indicador de Qualidade de Coleta e Disposição de Resíduos Sólidos na cidade $x$ no ano $y$;

$\mathrm{RS}_{1 \mathrm{x}, \mathrm{y}}=$ taxa de cobertura do serviço de coleta de resíduos na cidade $x$ no ano $y$;

$\mathrm{RS}_{2 \mathrm{x}, \mathrm{y}}=$ custo unitário médio do serviço de coleta na cidade $x$ no ano $y$; 
CRSmáx = custo unitário médio máximo estadual do serviço de coleta na cidade $x$ no ano $y$;

CRSmín = custo unitário médio mínimo estadual do serviço de coleta na cidade $x$ no ano $y$;

$\mathrm{RS}_{3 \mathrm{x}, \mathrm{y}}=$ taxa de recuperação de materiais recicláveis na cidade $x$ no ano $y$;

$\mathrm{RS}_{4 \mathrm{x}, \mathrm{y}}=$ custo unitário médio do serviço de varrição na cidade $x$ no ano $y$;

CVmáx = custo unitário médio máximo estadual do serviço de varrição na cidade $x$ no ano $y$;

CVmín $=$ custo unitário médio mínimo estadual do serviço de varrição na cidade $x$ no ano $y$; $\mathrm{RS}_{5 \mathrm{x}, \mathrm{y}}=$ disposição dos resíduos sólidos na cidade $x$ no ano $y$;

$\mathrm{RS}_{5 \mathrm{x}, \mathrm{y}}=0$, se a cidade tem lixão;

$\mathrm{RS}_{5 \mathrm{x}, \mathrm{y}}=75$, se a cidade tem aterro controlado;

$\mathrm{RS}_{5 \mathrm{x}, \mathrm{y}}=100$, se a cidade tem aterro sanitário;

Para o cálculo do IQRS foi adotado um peso 2,0 para taxa de recuperação de materiais recicláveis em relação à quantidade total coletada. Isso se justifica pelo fato de contribuir diretamente na conservação ambiental, pois o aumento de recuperação de materiais recicláveis diminui o gasto energético, a extração de recursos naturais e o volume de resíduos sólidos nos aterros sanitários. Além disso, ainda é algo recente nas considerações de saneamento básico e apenas adotado por algumas cidades.

\subsection{INDICADOR DE QUALIDADE DE DRENAGEM (IQD)}

O IQD engloba apenas o número de enchentes ou alagamentos, conforme SUDERHSA (2009b) nas cidades estudadas.

Existem poucos dados sobre enchentes, alagamentos e drenagem pluvial urbana. A maioria dessas informações fica arquivada em setores específicos das prefeituras. Como a aplicação do indicador está sendo feita em várias localidades, seria necessário um banco de dados estadual para que as cidades não precisassem ser 
visitadas para a coleta de informações. Entende-se que dados sobre riscos ambientais deveriam ser incluídos nesse indicador, caso estivessem disponíveis. Dessa forma, o IQD fica restrito a apenas uma variável.

Esse valor foi transformado em uma escala de 0 a 100, de acordo com os limites mostrados no Quadro 6, por interpolação simples.

Quadro 6. Variável para o cálculo do IQD e seu limite

\begin{tabular}{|c|c|c|c|}
\hline Códigos & Variáveis & $\begin{array}{c}\text { Limite para IQD } \\
\mathbf{1} \mathbf{1 0 0}\end{array}$ & $\begin{array}{c}\text { Limite para IQD } \\
\mathbf{0}\end{array}$ \\
\hline D & $\begin{array}{c}\text { Número anual de enchentes ou } \\
\text { alagamentos }\end{array}$ & 0 & 6 \\
\hline
\end{tabular}

O cálculo do IQD foi feito pelo percentual de enchentes ou alagamentos ocorridos no ano sobre o número de meses chuvosos, considerando como a pior situação o fato de haver, nesse período, uma enchente/alagamento por mês, como mostra a equação (7). Entretanto, se não há dados disponíveis de eventos críticos (enchente/alagamento), deve-se utilizar IQD igual a zero.

$$
\begin{aligned}
& I Q D_{x, y}=100-\left(100 \frac{D_{x, y}}{b}\right) \\
& \mathrm{IQD}_{\mathrm{x}, \mathrm{y}}=0 ; \text { se não há dados de } \mathrm{D}_{\mathrm{x}, \mathrm{y}}
\end{aligned}
$$

Com:

$\mathrm{x}=$ localidades, de 1 a 16 ;

$\mathrm{y}=$ tempo, de 2000 a 2008.

Onde:

$\mathrm{IQD}_{\mathrm{x}, \mathrm{y}}$ - Indicador de Qualidade de Drenagem de Águas Pluviais na cidade $x$ no ano $y$;

$\mathrm{D}_{\mathrm{x}, \mathrm{y}}=$ número de enchentes ou alagamentos na cidade $x$ no ano $y$;

$b=6$ (número de meses chuvosos, de forma geral e simplificada). 


\subsection{INDICADOR DE QUALIDADE DE SANEAMENTO BÁSICO URBANO (IQSBU)}

O IQSBU foi calculado como a média aritmética simples dos subindicadores IQAB, IQES, IQRS e IQD, conforme equação (8). Para que haja qualidade de saneamento básico, os quatro indicadores são de extrema importância.

$$
I Q S B U_{x, y}=\frac{I Q A B_{x, y}+I Q E S_{x, y}+I Q R S_{x, y}+I Q D_{x, y}}{4}
$$

Além do peso para a taxa de recicláveis, não foi discutida neste capítulo outra ponderação de pesos para as variáveis, pois a escolha de cada uma foi motivada pela existência dos dados disponíveis.

De 2000 a 2004 não há dados sobre drenagem, o que fez com que nesses anos a fórmula fosse apenas a média aritmética de IQAB, IQES e IQRS.

Para classificar os valores de IQSBU em função da qualidade do saneamento básico urbano, foi criada uma escala de valores, mostrada no Quadro 7. Em localidades cujos valores calculados ficaram entre 0 a 59 , considera-se que apresentam deficiência no saneamento básico urbano. Em localidades com valores de IQSBU acima de 60 a qualidade do saneamento está de boa a ótima.

Quadro 7. Valores do IQSBU e qualidade saúde urbana

\begin{tabular}{|c|c|}
\hline Valores do IQSBU & Qualidade de Saneamento Básico Urbano \\
\hline $80-100$ & Ótima \\
\hline $60-79,9$ & Boa \\
\hline $40-59,9$ & Regular \\
\hline $20-39,9$ & Ruim \\
\hline $0-19,9$ & Péssima \\
\hline
\end{tabular}

\section{RESULTADOS E DISCUSSÕES}

A realização do estudo para o desenvolvimento de um indicador de saneamento permitiu fazer as seguintes constatações quanto aos dados envolvidos: Apesar de o Ministério das Cidades manter o SNIS, há falta de vários dados. Essas lacunas prejudicam por parte das municipalidades. Esses os resultados 
de indicadores e as cidades que alimentam o sistema com suas informações são beneficiadas, pois seus subindicadores não ficam nulos.

Os itens que mais apresentam falhas são os de resíduos sólidos e os de drenagem. Essa carência faz com que os valores de IQRS e IQD sejam baixos.

A Figura 2 mostra os resultados obtidos de IQSBU para o ano de 2000, bem como as porcentagens de contribuição dos subindicadores.

Pode-se notar que em 2000 não há dados disponíveis sobre resíduos sólidos para as cidades estudadas. Isso prejudicou todas as cidades, sendo que o maior valor de IQSBU foi em Paranaguá $(30,2)$.



Figura 2. Resultados de IQSBU e porcentagens de contribuição dos subindicadores para 2000

Observa-se, também, que Paranaguá apresentou o maior valor de IQAB em relação às demais cidades. Isso ocorreu porque Paranaguá é a única cidade não servida pela Sanepar e, de 2000 a 2003, a Sanepar, em conjunto com o município, não repassou todos seus dados de qualidade para o sistema do Ministério das Cidades.

Dentre todas as cidades, Arapongas foi a mais prejudicada até 2004, pois não foram encontrados dados sobre saneamento básico.

Percebe-se pela Figura 3 que, em 2001, todos os valores de IQSBU (com exceção de Arapongas) foram maiores do que em 2000, embora não haja também 
dados sobre resíduos sólidos. A cidade de Paranaguá continuou com o maior valor de IQSBU $(47,3)$.



Figura 3. Resultados de IQSBU e porcentagens de contribuição dos subindicadores para 2001

Já em 2002, alguns municípios repassaram seus dados de resíduos sólidos para o banco do SNIS, como pode ser visualizado na Figura 4. Esses foram os que obtiveram os maiores resultados de IQSBU: Cascavel, Curitiba, Foz do Iguaçu, Guarapuava, Londrina, Maringá, Paranaguá e Toledo.



Figura 4. Resultados de IQSBU e porcentagens de contribuição dos subindicadores para 2002 
Toledo foi o município que em 2002 mais repassou seus dados para o SNIS e sua taxa de recuperação de materiais recicláveis foi a maior dentre todas as cidades $(21,9)$.

Além de Toledo, Cascavel, Curitiba e Londrina também se destacaram em 2002. As duas primeiras porque já utilizavam de aterro sanitário para disposição dos resíduos e a última pelos bons valores de IQES e IQRS.

Apucarana, Araucária, Campo Largo, Colombo, Pinhais, Ponta Grossa e São José dos Pinhais continuaram sem fornecer dados de resíduos sólidos.

A Figura 5 mostra os resultados para 2003. Se em 2002, apenas um município apresentou valor de IQSBU maior do que 50, em 2003 esse número já subiu para 5 municípios. Esse aumento aconteceu devido à existência de mais dados sobre abastecimento de água e sobre esgotamento sanitário.

Guarapuava atingiu um nível de boa qualidade de saneamento básico ( $>$ $60,0)$ em razão de alta taxa de tratamento de esgoto $(80,3)$ e alta taxa de recuperação de materiais recicláveis $(28,8)$.

Um resultado curioso é o fato de Londrina ter diminuído seu valor de IQRS devido à falta de repasse de informações.



Figura 5. Resultados de IQSBU e porcentagens de contribuição dos subindicadores para 2003

Os dados apresentados na Figura 5 mostram que Arapongas incluiu seus dados de saneamento no SNIS e, apesar de não ter disponível dados de resíduos sólidos, seu IQSBU ficou acima de 50,0. 
Em 2004, observa-se novamente um aumento geral de IQSBU em relação a 2003. Pode-se notar que à medida que os dados vão sendo disponibilizados, os valores de IQSBU vão traduzindo mais fielmente a realidade do saneamento básico das cidades (Figura 6).

Londrina e Curitiba apresentaram os maiores resultados de IQSBU em 2004, seguidas de Toledo, Foz do Iguaçu, Maringá e Cascavel.



Figura 6. Resultados de IQSBU e porcentagens de contribuição dos subindicadores para 2004

Verifica-se, pela Figura 7, a existência de dados de drenagem, apesar de falta de informações sobre resíduos sólidos.



Figura 7. Resultados de IQSBU e porcentagens de contribuição dos subindicadores para 2005 
Com relação ao abastecimento urbano, observa-se que Colombo tem o menor resultado. Apesar de ter o maior resultado para conformidade de água tratada, não existem dados sobre duração de paralisações, tarifa média de água, consumo médio per capita de água e índice de perdas na distribuição.

Guarapuava, Londrina e Toledo obtiveram os maiores resultados de IQSBU para 2005, ultrapassando o valor de 70,0.

O IQD foi alto para todas as cidades. Como esse subindicador é composto de apenas uma variável, a tendência é que seus resultados sejam altos.

Em 2006, os resultados foram os melhores de todo o período estudado (Figura 8). Quase metade das cidades tiveram IQSBU acima de 70,0 e onze cidades alcançaram uma boa qualidade de saneamento básico (> 60,0): Arapongas, Campo Largo, Cascavel, Curitiba, Foz do Iguaçu, Guarapuava, Londrina, Maringá, Pinhais, Ponta Grossa, São José dos Pinhais e Toledo.

Observa-se, também, que os resultados para IQAB foram maiores, no geral, em 2006 do que nos outros anos.

Ainda nota-se que, pela primeira vez, São José dos Pinhais apresentou dados de resíduos sólidos. Os demais municípios com IQRS igual a zero em 2005 permaneceram com esse valor em 2006.

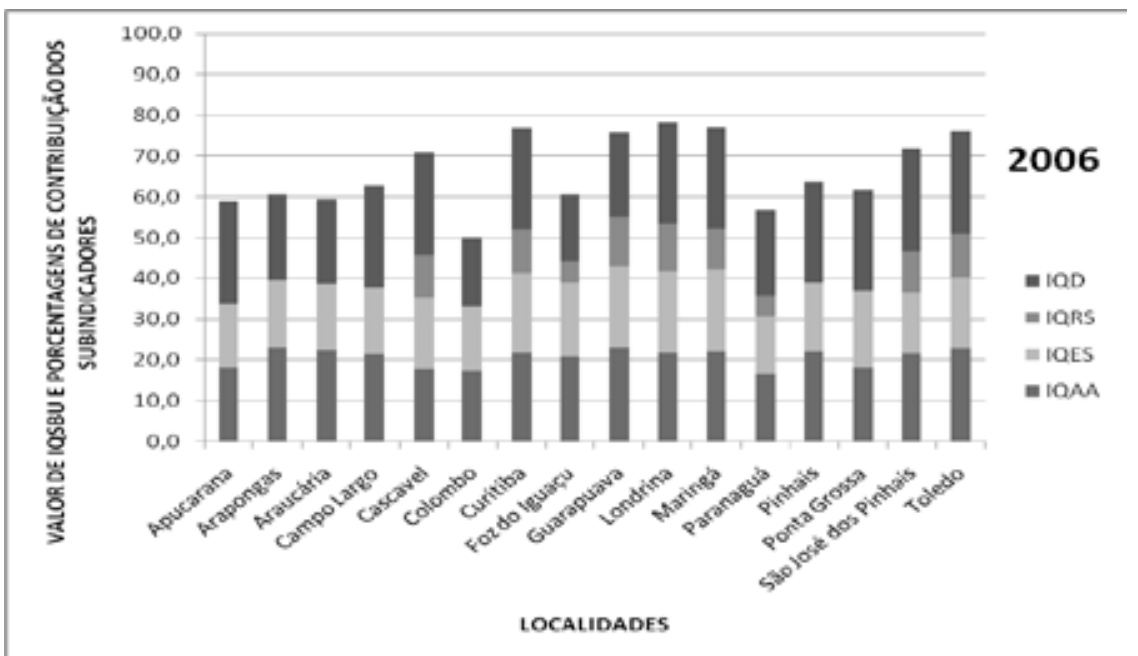

Figura 8. Resultados de IQSBU e porcentagens de contribuição dos subindicadores para 2006 
A Figura 9 mostra os resultados de IQSBU para 2007. Verifica-se que, no geral, são menores do que em 2006, pois apenas sete cidades obtiveram boa qualidade de saneamento.

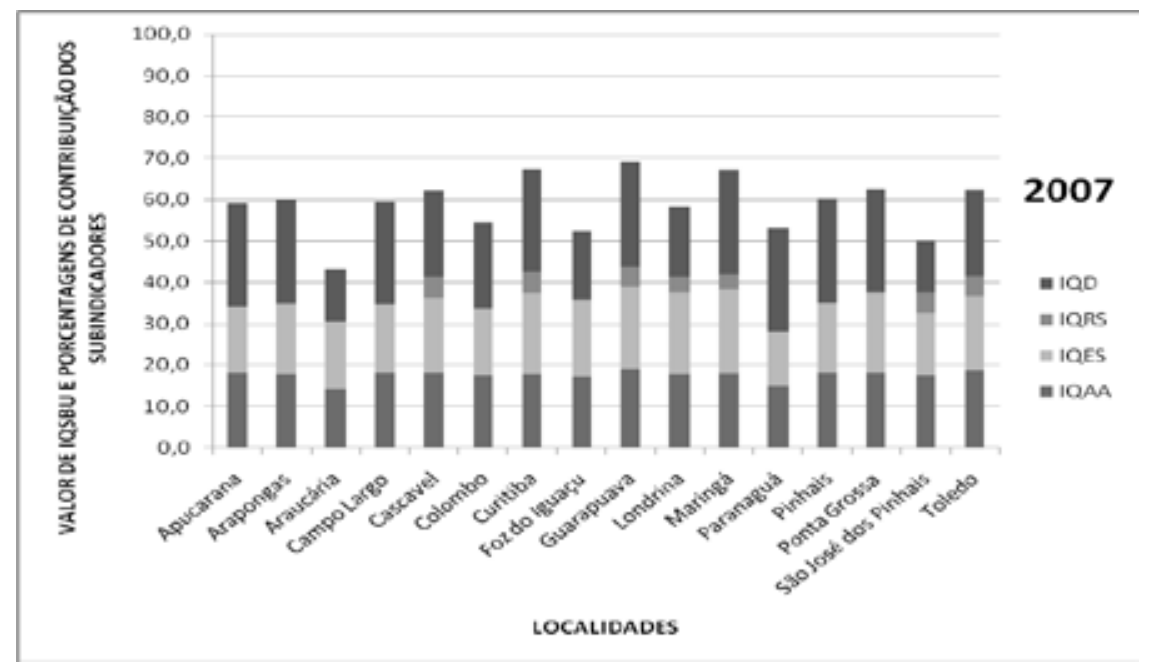

Figura 9. Resultados de IQSBU e porcentagens de contribuição dos subindicadores para 2007

A Tabela 1 mostra os valores de IQSBU para as localidades no período estudado. Nos primeiros dois anos Paranaguá obteve os maiores resultados, depois Toledo e, em seguida, houve uma alternância no primeiro lugar entre Guarapuava e Londrina.

Tabela 1. Valores de IQSBU para as localidades estudadas no período de 2000 a 2008

\begin{tabular}{lcccccccc}
\hline Localidades & $\mathbf{2 0 0 0}$ & $\mathbf{2 0 0 1}$ & $\mathbf{2 0 0 2}$ & $\mathbf{2 0 0 3}$ & $\mathbf{2 0 0 4}$ & $\mathbf{2 0 0 5}$ & $\mathbf{2 0 0 6}$ & $\mathbf{2 0 0 7}$ \\
\hline Apucarana & 9,4 & 32,3 & 27,2 & 41,5 & $\mathbf{4 3 , 7}$ & 57,1 & 59,0 & 59,1 \\
Arapongas & $\mathbf{0 , 0}$ & $\mathbf{0 , 0}$ & $\mathbf{0 , 0}$ & $\mathbf{0 , 0}$ & 51,6 & 59,3 & 60,5 & 59,9 \\
Araucária & 9,9 & 29,5 & 25,5 & 36,8 & 48,8 & 52,3 & 59,5 & $\mathbf{4 3 , 1}$ \\
Campo Largo & 10,2 & 28,4 & 27,5 & 24,7 & 50,1 & 62,0 & 62,8 & 59,6 \\
Cascavel & 11,6 & 33,9 & 46,1 & 57,7 & 60,6 & 63,3 & 70,8 & 62,1 \\
Colombo & 8,2 & 29,7 & 26,9 & 31,9 & 46,6 & $\mathbf{4 8 , 6}$ & $\mathbf{4 9 , 8}$ & 54,5 \\
Curitiba & 13,6 & 33,5 & 45,0 & 57,4 & 71,3 & 65,1 & 77,0 & 67,4 \\
& & & & & & & & \\
\hline
\end{tabular}


(conclusão)

\begin{tabular}{lllllllll} 
Foz do Iguaçu & 10,7 & 33,9 & 35,4 & 42,7 & 64,3 & 65,1 & 60,7 & 52,4 \\
Guarapuava & 12,7 & 36,3 & 38,1 & $\mathbf{6 9 , 2}$ & 61,9 & $\mathbf{7 5 , 6}$ & 76,0 & $\mathbf{6 9 , 0}$ \\
Londrina & 14,3 & 33,9 & 49,8 & 52,5 & $\mathbf{7 5 , 2}$ & $\mathbf{7 3 , 9}$ & $\mathbf{7 8 , 3}$ & 58,1 \\
Maringá & 13,4 & 36,5 & 38,2 & 47,0 & 62,7 & 62,0 & 77,1 & 67,1 \\
Paranaguá & $\mathbf{3 0 , 2}$ & $\mathbf{4 7 , 3}$ & 47,7 & 40,9 & 43,9 & 60,7 & 56,7 & 53,2 \\
Pinhais & 10,2 & 28,6 & 25,9 & 25,9 & 49,1 & 53,8 & 63,8 & 60,2 \\
Ponta Grossa & 12,4 & 32,7 & 30,1 & 46,2 & 52,9 & 64,3 & 61,9 & 62,6 \\
São José dos & 10,9 & 27,5 & 27,0 & 23,4 & 49,6 & 62,3 & 71,7 & 50,1 \\
Pinhais & 10,0 & 33,2 & $\mathbf{5 4 , 9}$ & 58,4 & 67,0 & 73,9 & 76,1 & 62,4 \\
\hline Toledo & & & & & & & &
\end{tabular}

Com relação aos menores valores, aparecem Arapongas nos quatro primeiros anos, seguida de Apucarana, Colombo e Araucária.

A Figura 10 mostra a evolução dos IQSBU e os limites das faixas de qualidade.

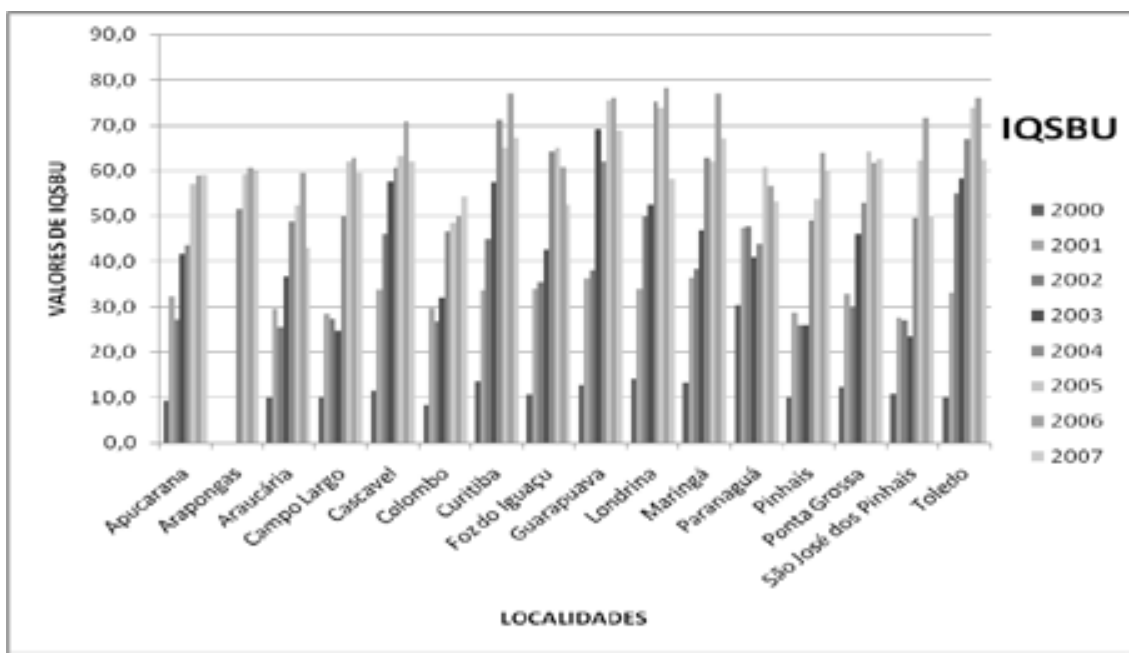

Figura 10. Evolução do IQSBU para as cidades de 2000 a 2007

Observa-se pela Figura 10 que, pelo método utilizado, nenhuma cidade atingiu ótima qualidade dos serviços de saneamento básico $(>80,0)$.

Houve um grande aumento percentual de 2000 a 2007 em todas as cidades, entretanto, não se pode afirmar que houve melhoria do saneamento, e sim, que houve maior repasse das informações para o SNIS. 
Da mesma forma, as cidades com os menores valores não são, necessariamente, os que apresentam piores condições sanitárias, mas são os que menos informações disponibilizaram.

De acordo com o estudo realizado, os anos que mais tiveram dados disponibilizados foram os de 2005 a 2007. Assim, os resultados para esses anos estão mais próximos da realidade do que os outros anos. Os piores resultados para esses três anos aconteceram em Colombo e Araucária, municípios da região metropolitana de Curitiba.

A maioria das cidades teve uma melhoria percentual de IQSBU, de 2005 a 2006. O maior aumento percentual ocorreu em Maringá (19,6\%), seguida de Pinhais $(15,6 \%)$ e Curitiba $(15,4 \%)$.

Foz do Iguaçu (7,3\%), Paranaguá $(7,1 \%)$ e Ponta Grossa (3,9\%) tiveram quedas percentuais de 2005 a 2006.

Com relação ao período de 2006 a 2007, ocorreu o contrário: a maioria das cidades teve uma diminuição percentual do IQSBU. São José dos Pinhais $(43,2 \%)$, Araucária $(48,1 \%)$ e Londrina (34,9\%) tiveram as maiores quedas percentuais. Colombo $(8,6 \%)$, Ponta Grossa $(1,1 \%)$ e Apucarana $(0,2 \%)$ tiveram pequenos aumentos percentuais de IQSBU.

As Figuras 11 e 12 mostram os resultados de IQSBU para o Paraná em 2000 e 2007 .

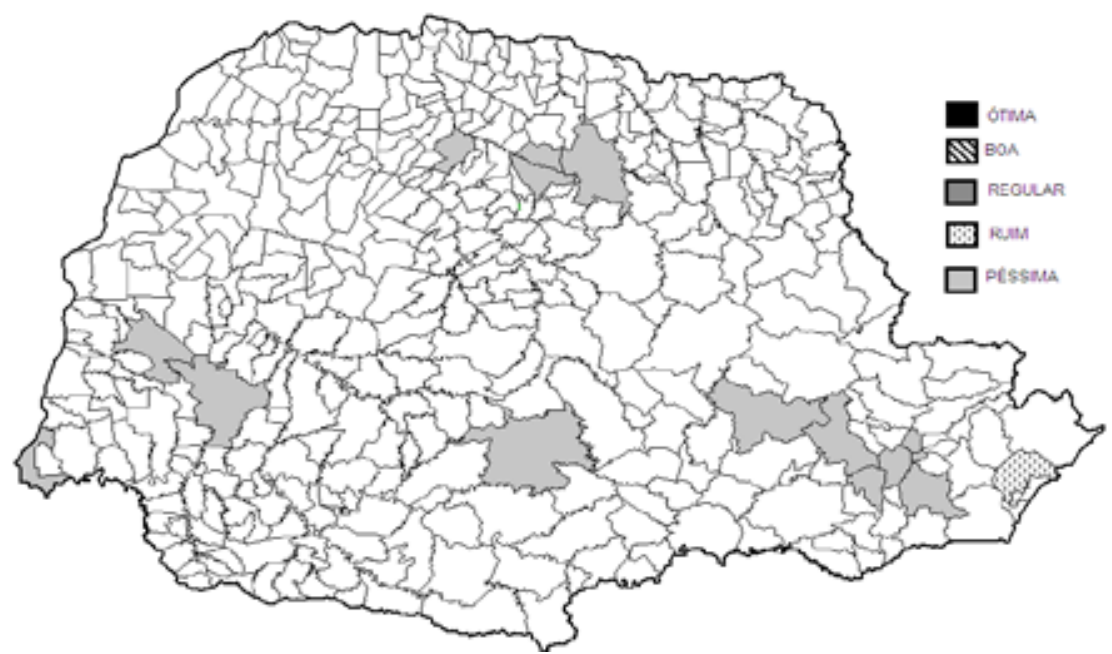

Figura 11. Resultado de IQSBU para o Paraná em 2000 


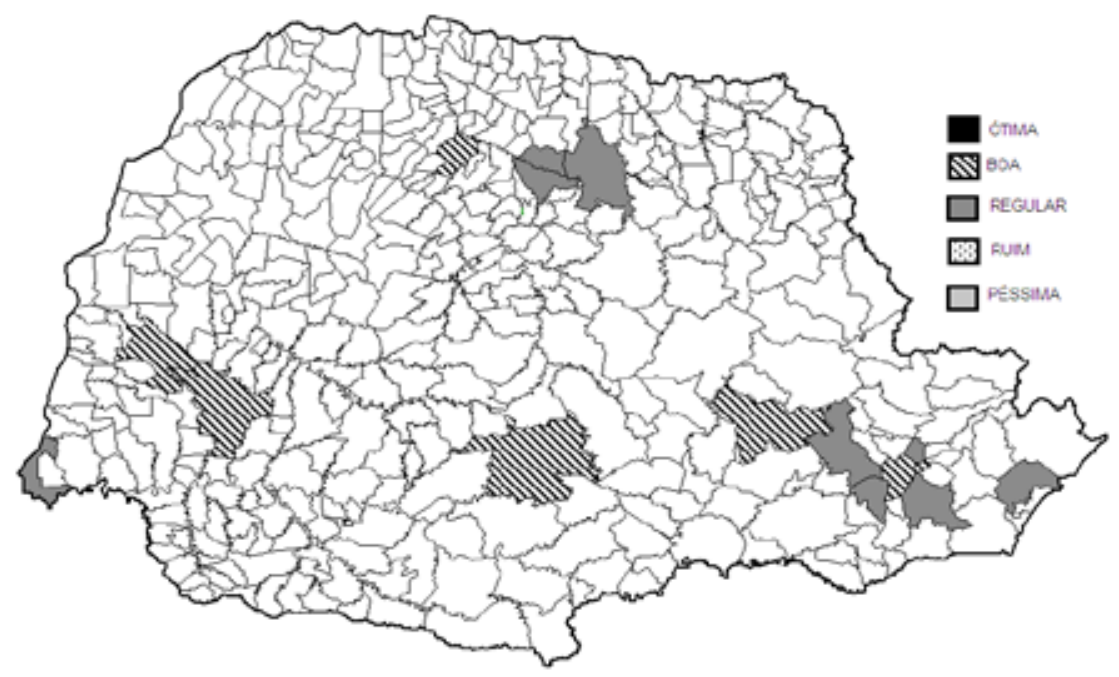

Figura 12. Resultado de IQSBU para o Paraná em 2007

\section{CONSIDERAÇÕES FINAIS E CONCLUSÕES}

Após o término desse estudo, podem-se obter algumas conclusões, sendo a principal delas a constatação de falta de informações sobre o saneamento ambiental urbano, em especial sobre resíduos sólidos e drenagem. Isso implica em dificuldades na gestão e no planejamento urbano. Recomenda-se que o IQD seja aprimorado e ampliado em trabalhos futuros. Também deve ser aprofundada a metodologia do IQSBU, pois há estreita relação desse tema com a saúde pública e preservação ambiental.

Se todos os dados fossem disponibilizados no SNIS, o cálculo dos indicadores seria mais fiel à realidade sanitária das cidades. Essas lacunas prejudicamos resultados de indicadores e as cidades que alimentam o sistema com suas informações são beneficiadas, pois seus subindicadores não ficam nulos. Dessa forma, é possível verificar e sanar as deficiências de infraestrutura e serviços, manter o ambiente preservado e reparar a degradação ambiental.

Com relação aos resultados, conclui-se que, no geral, as cidades apresentaram uma boa qualidade de saneamento básico, em pelo menos um ano do 
período. Observou-se, entretanto, que nenhuma cidade atingiu ótima qualidade dos serviços de saneamento básico $(>80,0)$. As cidades que não tiveram nenhum valor de IQSBU acima de 60 foram: Apucarana, Araucária e Colombo.

$\mathrm{O}$ ano com melhores resultados de IQSBU e, consequentemente, de maior repasse das informações pelos municípios ao sistema foi 2006. Nesse ano, o maior valor encontrado para IQSBU foi para Londrina $(78,3)$ e o menor foi para Colombo $(49,8)$.

\section{REFERÊNCIAS}

BELLEN, H. M. 2006. Indicadores de sustentabilidade: uma análise comparativa. 2.ed. Rio de Janeiro: Fundação Getúlio Vargas. 256p.

BOLLMANN, H. A.; MAIA, N. B.; BARRELLA, W. Indicadores ambientais: conceitos e aplicações. São Paulo: EDUC, 2001.

BRASIL. Ministério das Cidades. Elementos para organização da coleta seletiva e projeto dos galpóes de triagem. Brasília: Ministério das Cidades, 2008.

DEAKIN, M. et al. Sustainable urban development: the environment assessment methods. Routledge: New York, 2007. Vol. 2, p. 308.

HOLDEN, M. Urban indicators and the integrative ideals of cities. Cities. n. 23. v. 3. p. $170-183,2006$.

INSTITUTO BRASILEIRO DE GEOGRAFIA E ESTATÍSTICA. IBGE. PNSB 2008: Abastecimento de água chega a 99,4\% dos municípios, coleta de lixo a 100\%, e rede de esgoto a 55,2\%. 2011b. Disponível em: < http://www.ibge.gov.br/home/ presidencia/noticias/noticia_visualiza.php?id_noticia $=1691 \& i d$ _pagina $=1>$. Acesso em: 11 fev. 2011.

ICLEI. Conselho Internacional para Iniciativas Ambientais Locais. 2nd World Congress on Cities and Adaptation to Climate Change. 2011. Disponível em: < http://resilient-cities.iclei.org/> . Acesso em: 15 abr. 2011. 
MAIA, N. B.; MARTOS, H. L.; BARRELLA, W. (Org.). Indicadores ambientais: conceitos e aplicações. São Paulo: EDUC, 2001.

MINISTÉRIO DAS CIDADES. Secretaria Nacional de Saneamento Ambiental. Sistema Nacional de Informações sobre Saneamento. Brasília: Ministério das Cidades, Secretaria Nacional de Saneamento Ambiental, Programa de Modernização do Setor Saneamento, 2009. (Série histórica 6). Disponível em: <www.snis.gov.br >. Acesso em: 05 ago. 2009.

ORGANIZAÇÃO DE COOPERATIVISMO E DESENVOLVIMENTO ECONÔMICO. OCDE. Organization for economic cooperation and development: core set of indicators for environmental performance reviews; a synthesis report by the group on the state of the environment. Paris: OCDE, 1993.

SANEPAR. Companhia de Saneamento do Paraná. Relatório Anual da Qualidade da Água. Curitiba: Sanepar, 2005.

SANEPAR. Companhia de Saneamento do Paraná. Relatório Anual da Qualidade da Água. Curitiba: Sanepar, 2006.

SANEPAR. Companhia de Saneamento do Paraná. Relatório Anual da Qualidade da Água. Curitiba: Sanepar, 2007.

SANEPAR. Companhia de Saneamento do Paraná. Relatório Anual da Qualidade da Água. Curitiba: Sanepar, 2008.

SICHE, R.; AGOSTINHO, F.; ORTEGA, E.; ROMEIRO, A. Índices versus indicadores: precisões conceituais na discussão da sustentabilidade de países. Ambiente \& Sociedade, Campinas, v. X, n. 2, p. 137-148, 2007.

SUPERINTENDÊNCIA DE DESENVOLVIMENTO DE RECURSOS HÍDRICOS E SANEAMENTO AMBIENTAL. SUDERHSA. Destinação final: resíduos sólidos urbanos - Paraná. 2009a. Disponível em: < http://www.suderhsa.pr.gov.br/meioambiente/ arquivos/File/suderhsa/Dest_Res_Urbanos_PR_jUL2008.pdf $>$. Acesso em: 15 ago. 2009. 
SUPERINTENDÊNCIA DE DESENVOLVIMENTO DE RECURSOS HÍDRICOS E SANEAMENTO AMBIENTAL. SUDERHSA. Elaboração do Plano Estadual de Recursos Hídricos: Produto 1.2 - Parte D: Avaliação das disponibilidades hídricas, eventos críticos e monitoramento do uso de recursos hídricos. 2009b. Disponível em: <http://www.suderhsa.pr.gov.br/modules/conteudo/conteudo. php?conteudo $=108>$. Acesso em: 15 ago. 2009 .

TUCCI, C. E. M. Gerenciamento da drenagem urbana. Revista Brasileira de Recursos Hídricos, v. 7. n. 1, 2002.

Recebido em: 10 de fevereiro de 2014 Aceito em: 16 de fevereiro de 2014 